\title{
A Advocacia em Saúde como uma Estratégia Para a Promoção da Saúde
}

\author{
Regina Célia Canel(*) \\ Cláudio Gastão Junqueira de Castro ${ }^{(* *)}$
}

\section{RESUMO}

O conceito de Promoção da Saúde vem sendo desenvolvido há aproximadamente trinta anos e já está sedimentado. Atualmente, torna-se muito importante elaborar estratégias para sua implementação. O presente artigo é uma reflexão sobre a prática da Advocacia em Saúde enquanto estratégia para a implementação dos princípios e diretrizes da Promoção da Saúde. O ponto de partida é o questionamento sobre como conciliar os aspectos conceituais e metodológicos da Promoção da Saúde, considerados dentro de nossa realidade. A conclusão principal aponta para uma proposta de desenvolvimento de uma "Advocacia em Promoção da Saúde".

\section{Palavras-chave}

Advocacia em Saúde; Políticas Públicas; Promoção da Saúde.

\section{ABSTRACT}

The concept of Health Promotion has been developed approximately thirty years ago and it is already well constructed. Currently, it is very important to develop strategies for its implementation. This article is a reflection on the

(*) Psicóloga, Mestre em Saúde Pública, Especialista em Saúde Mental e em Administração Pública. E-mail: <reginacanel@usp.br>

$\left.{ }^{* *}\right)$ Professor Doutor do Departamento de Prática de Saúde Pública da Faculdade de Saúde Pública, da Universidade de São Paulo. E-mail: <claudiog@usp.br>. 
practice of the Health Advocacy as a strategy for the implementation of the principles and guidelines of the Health Promotion. This works starts questioning how to conciliate conceptual and methodological aspects of the Health Promotion, considering our reality. The main conclusion points to a proposal to develop a "Health Promotion Advocacy.

\section{Keywords}

Health Advocacy; Health Promotion; Public Politics.

\section{PROMOÇÃO DA SAÚDE}

O conceito de Promoção da Saúde (PS) foi inicialmente introduzido em 1945 por $H$. Sigerist, no Canadá, como uma área da Medicina. Para essa área, Sigerist atribuiu as seguintes finalidades: a promoção da saúde, envolvendo fatores socioambientais, medidas políticas e trabalho comunitário; a prevenção e tratamento de doenças; a reabilitação.

Seguiu-se, em 1974, o Informe Lalonde, também do Canadá, como uma proposta de ampliação do campo de atuação da Saúde Pública, acrescentando às ações médico-assistenciais ações preventivas e programas educativos, objetivando mudanças comportamentais e de estilo de vida. $\mathrm{O}$ enfoque, entretanto, era sobre o indivíduo, considerado como responsável exclusivo por sua saúde e seu estilo de vida.

No ambiente internacional, o marco inicial da PS foi a Conferência de Alma-Ata (1978). A esta, seguiram-se outras conferências e respectivas Cartas e Declarações: Ottawa, 1986; Adelaide, 1988; Sundsvall, 1991; Bogotá, 1992; Jacarta, 1997; Rede de Megapaíses, 1998; México, 2000; Bangkok, 2005. (1)

Nesses documentos, evidencia-se um novo entendimento do conceito de saúde e de seus fatores determinantes, constituindo-se a "nova promoção da saúde"(2). Esta concepção propõe que se atue sobre tais determinantes, ou seja, sobre as condições de vida da população, mediante ações intersetoriais (que envolvem, por exemplo, meio ambiente, educação, habitação).

(1) BRASIL. Ministério da Saúde. Promoção da Saúde: Declaração de Alma-Ata, Carta de Ottawa, Declaração de Adelaide, Declaração de Sundsvall, Declaração de Santafé de Bogotá, Declaração de Jacarta, Rede de Megapaíses e Declaração de México. Brasília, 2001; UNESCAP — United Nations Economic and Social Commission for Asia and the Pacific. Disponível em: <www.unescap.org/ esid/hds/whatsnew/6GCHP_1.asp>. Acesso em: nov. 2005.

(2) SÍCOLI, J. L.; NASCIMENTO, P. R. Promoção da saúde: concepções, princípios e operacionalização. Interface - Comunicação, Saúde, Educação, v. 7, n. 12, p. 101-22, 2003. 
A síntese das Cartas e Declarações mostra que a saúde resulta de um conjunto de fatores individuais e coletivos (sociais, econômicos, políticos, étnicos, religiosos, culturais, psicológicos, do trabalho, biológicos, ambientais), interagindo num processo dinâmico. As Cartas consideram a saúde como um direito humano fundamental e essencial para o desenvolvimento pessoal, social e econômico ${ }^{(3)}$. Sua manutenção deve ser o principal investimento social dos governos.

Os documentos reforçam a idéia de que o processo saúde-doença não é apenas linear (resultante de causa-efeito) e nem tão pouco restrito ao ponto de vista biomédico, mas deve ser percebido dentro de um novo modelo (holístico), no qual as metas da saúde são atingidas juntamente com a melhoria da qualidade de vida, a justiça social e o desenvolvimento sustentado. A participação comunitária é fundamental e os profissionais de saúde devem assumir a tarefa de estimular e fortalecer a atuação da população nessas questões, instrumentalizando os cidadãos (indivíduos, grupos) para que seja exercido o direito à saúde. Tudo isso constitui uma nova cultura, ou um novo paradigma, da saúde.

As Cartas mencionam aspectos essenciais relativos à PS, como os seguintes:

1) necessidade de políticas públicas saudáveis: o principal propósito destas políticas é a criação de ambientes físicos e sociais favoráveis à saúde, com um novo entendimento de desenvolvimento social e do papel do Estado, esse centrado nas necessidades dos cidadãos, na eqüidade, na intersetorialidade, na democratização do poder, na sustentabilidade;

2) atenção ao meio ambiente: por meio de estratégias como aliança entre as áreas da saúde e do ambiente, tendo em vista sua interdependência;

3) eqüidade: prioridade de alocação, dimensionamento e distribuição de recursos e serviços de saúde aos grupos sociais menos privilegiados e mais vulneráveis;

4) participação popular: implementada por meio da educação para a saúde, da informação, do direito de voz das pessoas e das comunidades (empoderamento), no planejamento e na execução dos cuidados de saúde;

5) reorientação dos serviços de saúde: utilizando critérios de PS e fomentando a preparação dos recursos humanos;

(3) BRASIL. Ministério da Saúde. Promoção da Saúde: Declaração de Alma-Ata, Carta de Ottawa, Declaração de Adelaide, Declaração de Sundsvall, Declaração de Santafé de Bogotá, Declaração de Jacarta, Rede de Megapaíses e Declaração de México, cit., p.43. 
6) parceria entre setor público e setor privado: ação coordenada e alianças entre governo, setor privado e terceiro setor, compondo parcerias em prol da saúde;

7) enfoque multissetorial: governo e setores sociais são igualmente responsáveis quanto às conseqüências de suas decisões e ações e quanto aos efeitos e repercussões de seus objetivos, estratégias, atividades e recursos nos demais setores. Todos os setores - mesmo aqueles que, aparentemente, não estão diretamente implicados devem assumir a saúde como meta;

8) necessidade de um esforço internacional em direção à PS.

A Organização Mundial da Saúde (OMS) introduziu oficialmente o conceito de PS em 1984(4), afirmando que ele envolve a população como um todo no contexto cotidiano, em lugar de concentrar a atenção em grupos de risco e doenças específicas. Em 1998, a OMS definiu sete princípios que devem nortear programas, políticas e planejamentos em $\mathrm{PS}^{(5)}$, reforçando as concepções antes formuladas nesse sentido, e enfatizando e ampliando com as seguintes propostas, além daquelas:

1) ações multiestratégicas: por meio da combinação de métodos e abordagens de diferentes disciplinas;

2) sustentabilidade: envolvendo interação entre diferentes variáveis, tais como equilíbrio ambiental, qualidade de vida, justiça social e capacidade de suporte (econômico-financeiro e institucional). As iniciativas em PS devem estar de acordo com esse princípio, uma vez que, em geral, tratam-se de iniciativas de natureza complexa que envolvem processos de transformação coletiva a médio e longo prazos, com articulações entre o local e o global.

Importante notar que, para implementar políticas de PS, de acordo com os princípios acima descritos, devem ser incrementados os exercícios da democracia e da cidadania (este último entendido, neste contexto, como luta por direitos e por políticas públicas saudáveis).

\section{ADVOCACIA EM SAÚDE}

No século $X X$, reconheceu-se a interdependência entre a saúde e as condições de trabalho, o que levou à instituição da previdência social e ao desenvolvimento da prevenção e da proteção sanitárias. Aos poucos, foi se

(4) WORLD HEALTH. Organization. Health promotion evaluation. Copenhagen, 1984.

(5) Id. Health promotion evaluation: recommendations to policymakers. Copenhagen, 1998. 
cristalizando a noção do direito sanitário(6), entendido como um sistema normativo. De fato, o Direito Sanitário se orienta pelos princípios da supremacia do interesse público sobre o particular e da indisponibilidade do interesse público. Ele trata tanto do direito à saúde (enquanto direito humano universal), como do direito da saúde pública, estabelecendo normas jurídicas na área. Há uma grande intersecção entre o direito sanitário e aquela atividade chamada "Advocacia em Saúde"(7).

De modo geral, entende-se por "advocacia" (advocacy) um processo de reivindicação de direitos, ou a atividade de um grupo, visando a influir na definição ou na implementação de uma política pública. A suposição básica é que as pessoas têm direitos fundamentais que são exigíveis por meio de procedimentos legislativos, administrativos ou judiciais.

Os direitos básicos podem até estar confirmados por normas legais; mas, quando falhas institucionais limitam seu exercício, resultam problemas que produzem danos individuais ou coletivos. No sentido aqui utilizado, a advocacia é dirigida a sanar estes problemas. Sua ação tem conotação política e se justifica quando há distribuição inadequada de autoridade ou de recursos ou quando há falhas no atendimento dos direitos (por falta de leis ou normas específicas, ou por omissões institucionais e administrativas).

A advocacia, como aqui entendida, é diferente da advocacia tradicional, isto é, da prestação de serviços profissionais por um bacharel em Direito. $\mathrm{Na}$ advocacia tradicional, os interesses envolvidos costumam ser individuais, privados, coletivos ou de grupos, sendo que há ainda aqueles públicos ou do Estado. Em todos esses casos, os titulares estão bem caracterizados. Mas, há outros e importantes interesses, chamados difusos, que não se referem a pessoas específicas: são aqueles mais amplos que os públicos, os quais podem ser de toda a humanidade, podendo até referir-se às gerações futuras.

É difícil identificar com precisão os titulares dos interesses difusos e daí decorrem as dificuldades de defesa destes direitos e de viabilizar a tutela de seus interesses. Em tais casos, a advocacia (no sentido de advocacy) é o procedimento eficaz para a proteção dos direitos difusos. O advogado, na advocacy, pode ser qualquer pessoa, não necessariamente um bacharel(8).

Quando o interesse envolvido é o direito à saúde, então a advocacia se especializa em Advocacia em Saúde. Ela se baseia nos esforços organizados de indivíduos e de grupos, para influenciar governo, empresas, administração

(6) DALLARI, S. G. Direito sanitário. In: MANUAL do Curso de Especialização em Direito Sanitário à Distância. Rio de Janeiro, 2002. p. 47-72.

(7) Id. Ibid.

(8) DALLARI, S. G. et al. Advocacia em saúde no Brasil contemporâneo. Revista de Saúde Pública, São Paulo, v. 30, n. 6, p. 592-601, 1996. 
pública, de modo que esses órgãos se tornem mais sensíveis às necessidades dos cidadãos - por exemplo, mediante o estabelecimento de políticas públicas que garantam a justiça social e o acesso universal à saúde.

A saúde pública não é estritamente um interesse difuso; mas, como ela depende de políticas sociais e econômicas, torna-se necessário, ao advogar em saúde, considerar também a defesa destes interesses. Quando o direito à saúde é específico à Saúde Pública, este faz parte do direito administrativo ${ }^{(9)}$.

A prática tem mostrado que a advocacia é mais eficaz quando concentrada em assuntos específicos, não genéricos; o problema deve ser muito bem identificado e caracterizado, para que se possa achar, mais eficazmente, uma solução apropriada. Entre as ações básicas da Advocacia em Saúde estão a alteração de leis, o monitoramento do legislativo, a elaboração e implementação de orçamentos, o acompanhamento de processos administrativos.

O processo de Advocacia em Saúde se desenvolve em diversas etapas, a saber:

1) estudo do problema específico, identificação dos direitos não atendidos, da legislação pertinente, das possíveis ações corretivas institucionais e das barreiras que podem ser encontradas;

2) coleta e apresentação dos dados do problema, localizando-os no tempo e no espaço;

3) elaboração das estratégias plausíveis, em função da situação política do momento, do interlocutor escolhido, de sua localização. Dependendo da situação, possíveis estratégias são: a) caso individual; b) ação civil pública; c) iniciativa popular e audiência pública; d) advocacia administrativa dirigida às agências governamentais. A escolha da estratégia depende também da localização do problema: execução de ações, de políticas públicas, de aspectos legislativos ou normativos ou da não observância de lei existente;

4) apresentação das estratégias ao beneficiário, e escolha — por parte do advogado, em conjunto com o beneficiário — da estratégia considerada melhor e das estratégias alternativas;

5) aplicação da estratégia escolhida;

6) avaliação contínua dos resultados e eventual reformulação da estratégia.

Na listagem acima, ressalte-se que "advogado" é o cidadão que conduz a ação de Advocacia em Saúde; ainda que ele não seja um bacharel, durante o processo ele passa a ser, temporariamente, um cidadão-advogado.

(9) DI PIETRO, M. S. Z. Direito administrativo. São Paulo: Atlas, 2000. p. 481-499. 
Organizações não governamentais e grupos de pressão (lobbies) também são exemplos do papel do advogado, que podem ser considerados atores propositivos.

Com freqüência, as ações de Advocacia em Saúde são dirigidas aos órgãos do Estado - considerando que a atuação dos poderes públicos é fundamental para alcançar o direito à saúde - e principalmente ao Legislativo. Outras ações podem influenciar os Conselhos de Saúde, e/ou eventualmente receber apoio do Ministério Público.

\section{A ADVOCACIA EM SAÚDE COMO UMA ESTRATÉGIA PARA A PROMOÇÃO DA SAÚDE}

No Brasil, começa-se a falar de PS com mais ênfase a partir da $8^{\circ}$ Conferência Nacional de Saúde de 1986, que politiza a questão da Saúde na preparação da Constituinte (Assembléia Nacional Constituinte de 1987, que formulou a Constituição Brasileira de 1988). Nesse mesmo ano, tem lugar a Primeira Conferência Internacional sobre Promoção da Saúde (Ottawa, Canadá), cujo documento pode ser considerado o mais incisivo quanto aos princípios da PS ${ }^{(10)}$.

Na Constituição de 1988, são colocados os princípios do Sistema Único de Saúde (SUS). A Carta Magna prevê que o Estado regulamente, fiscalize e controle os serviços de saúde. O art. 196 reconhece que a saúde é um direito de todos e dever do Estado, "garantido mediante políticas sociais e econômicas que visem a redução do risco de doenças e de outros agravos e ao acesso universal e igualitário às ações e serviços para sua promoção, proteção e recuperação".

Quando se descreve um sistema de saúde, é necessário considerar sua estruturação em três aspectos: o espaço jurídico, base legal para a política pública; o espaço institucional, modelo de gestão e arcabouço organizacional para efetivar princípios e diretrizes e o espaço operativo, modelo de atenção e processo de prestação de serviço ${ }^{(11)}$.

A nova Constituição formalizou a existência do espaço jurídico para uma nova política pública de saúde e para um novo sistema de saúde (SUS), dentro de um processo de redemocratização do Estado e da própria área da Saúde, mediante a participação e controle social.

(10) BRASIL. Ministério da Saúde. Promoção da Saúde: Declaração de Alma-Ata, Carta de Ottawa, Declaração de Adelaide, Declaração de Sundsvall, Declaração de Santafé de Bogotá, Declaração de Jacarta, Rede de Megapaíses e Declaração de México, cit.

(11) CONASEMS - Conselho Nacional de Secretários Municipais de Saúde. Pacto de saúde, Brasília, 2006. Disponível em: <www.conasems.org.br/files/dia27/ Of2ConstruindoMinisterioSaude02.pdf>. Acesso: maio 2007. 
Quanto ao espaço institucional, a nova política de saúde tem como diretrizes para o SUS a descentralização, a regionalização (os sistemas locais de saúde, sendo os municípios a base do sistema) e a direção única em cada esfera do governo (municipal, estadual e federal). Isso cria condições para ampliar a participação e o controle por parte da sociedade, como foi estabelecido na Lei n. 8.142/90.

Com relação ao espaço operativo, o SUS tem como diretrizes:

a) que as ações de saúde garantam a universalidade da cobertura (que estejam disponíveis e correspondam às necessidades locais) e do acesso (levando-se em conta os aspectos geográficos e financeiro, a cultura local, além de ser resolutivo e permanente);

b) a integralidade quanto à natureza das ações e serviços (prevenção primária, secundária e terciária) e pela integração com outros setores, através de parcerias, convênios, entre outros;

c) a base do sistema e sua funcionalidade estão assentadas na atenção básica e na resolução das questões de saúde no nível local (nos municípios);

d) o foco da atenção está na família e na orientação comunitária, sendo que, no Brasil, a estratégia da saúde da família é um componente forte da atenção básica em saúde.

A PS é uma concepção pela qual a saúde não se define em termos de ausência ou presença de doença, mas é dirigida ao entendimento dos determinantes da saúde e da doença e à atuação sobre eles. Esses determinantes referem-se tanto a fatores diretamente relacionados ao comportamento e ao controle direto pelos indivíduos (por exemplo, estilo de vida), como também àqueles que escapam a esse controle (por exemplo, fatores socioeconômicos, tipo e qualidade dos serviços de saúde disponíveis). Governos democráticos e participação social são pressupostos para a viabilização de projetos em $\mathrm{PS}^{(12)}$.

Assim, a consolidação da PS demanda uma visão interdisciplinar e de diálogo democrático e participativo entre os diversos atores e nos diversos contextos envolvidos (instituições públicas, privadas, governamentais e não governamentais, e cidadãos no geral), o que ultrapassa os marcos do setor específico da Saúde.

O novo paradigma "Promoção da Saúde" é uma nova maneira de interpretar as necessidades e as ações de saúde, passando de uma perspectiva "biológica, mecanicista, individual, específica", para uma perspectiva

(12) MELLO, D. A. Reflexões sobre a promoção de saúde no contexto do Brasil. Cadernos de Saúde Pública, v. 16, n. 4, 2000. 
"contextual, histórica, coletiva, ampla"(13). E é também uma nova maneira de agir em saúde: não se trata apenas de controlar fatores de risco e executar ações médico-curativas, mas também de desenvolver novos modelos de atenção à saúde e ações políticas voltadas para a saúde coletiva. No Brasil, essa nova maneira de agir em saúde é garantida pela Constituição e está nos princípios do SUS, o qual, por sua vez, está claramente assentado em princípios da PS.

Com isso, passa-se do aspecto conceitual da PS para o aspecto metodológico: práticas, planos de ação, estratégias, formas de intervenção e outros instrumentos $^{(14)}$. E é justamente no aspecto metodológico que se encontram as maiores dificuldades na condução e na realização desse novo paradigma, como, por exemplo, na eficácia e na efetividade dos programas de $P S^{(15)}$.

Como então superar essas dificuldades, de forma que os princípios da PS - previstos nas Cartas e Declarações das Conferências de Promoção da Saúde e pela OMS - sejam transformados em práticas consistentes e duradouras, materializadas no SUS? E, principalmente, como tornar eficaz a participação popular na formulação de políticas públicas locais e na elaboração, execução, avaliação e monitoramento de projetos e programas de intervenção?

Algumas estratégias são fundamentais quanto à eficácia e efetividade de ações e programas de PS. Dentre elas, podem-se destacar a intersetorialidade, o desenvolvimento de parcerias (vínculos de colaboração e de intercâmbio), a educação em saúde e a defesa da causa da saúde (Advocacia em Saúde).

Essa última estratégia (Advocacia em Saúde) é considerada pela União Internacional de Promoção da Saúde e Educação para a Saúde (UIPES) como uma de suas áreas prioritárias em suas Orientações Estratégicas (para o período de 1999 a 2004). De acordo com a UIPES, a Advocacia visa conquistar o apoio e o compromisso político de instâncias decisórias, a aceitação social e os espaços de discussão e de atuação em favor da saúde. Dito de outra forma, a Advocacia em Saúde é constituída por ações de indivíduos ou de grupos organizados - os chamados atores sociais - que procuram influir sobre autoridades e sobre particulares, para reivindicar direitos na área da saúde, principalmente em benefício da parcela da população menos favorecida.

(13) PEREIRA, I. M. T. B. et al. Promoção da saúde e educação em saúde: uma parceria saudável. O Mundo da Saúde, ano 24, v.2 4, n. 1, p. 39-44, 2000.

(14) CERQUEIRA, M. T. Promoción de la salud y educación para la salud: retos y perspectivas. In: ORGANIZACIÓN MUNDIAL DE LA SALUD. La promoción de la salud y la educación para la salud en América Latina: un análisis sectorial. Genebra: Editorial de La Universidad de Puerto Rico, 1997. p. 7-48.

(15) UIPES - União Internacional de Promoção da Saúde e Educação para a Saúde. Editorial. Boletim, ano 7, n. 9, 2005. 
Ainda de acordo com a UIPES, é importante que os profissionais da saúde - enquanto promotores da saúde — se percebam e se preparem como atores privilegiados da Advocacia em Saúde. Nesse papel, eles podem dar assessoria para as comunidades organizadas, aos órgãos do governo e às organizações não governamentais, além de disseminar informações sobre experiências em Advocacia em Saúde e, finalmente, ajudar a conscientizar o público e os políticos quanto às questões de saúde ${ }^{(16)}$.

A Carta de Bangkok - elaborada durante a VI Conferência Mundial sobre a Promoção da Saúde, em agosto de 2005 - enfatiza as estratégias para a PS num mundo globalizado, destacando-se ${ }^{(17)}$ :

- advogar para a saúde, com base em direitos humanos e em solidariedade;

- investir em políticas sustentáveis, ações e infra-estruturas para fomentar os determinantes da saúde;

- construir capacitação para o desenvolvimento das políticas, da liderança, da prática da PS, da transferência do conhecimento e da pesquisa, e da "alfabetização" em saúde;

— produzir leis e regulamentos que assegurem um alto nível de proteção contra danos e que proporcionem igualdade de oportunidade para a saúde e o bem-estar para todas as pessoas;

- montar parcerias entre organizações públicas, privadas, não governamentais e a sociedade civil, para criar ações sustentáveis.

As ações da Advocacia em Saúde são realizadas de várias formas, através dos meios de comunicação, por grupos de pressão política, entre outros, com o objetivo de favorecer a conscientização - por parte dos diversos atores - dos determinantes da saúde, e ainda de interferir sobre a formulação e a implementação de políticas públicas.

Assim, a Advocacia em Saúde é uma estratégia importante para promover o empoderamento, por parte da população, em relação às questões de saúde, não só para fazer valer o que já está legitimado, como também para tornar legítimo tudo o que é identificado como necessidade de saúde, justiça e inclusão social.

Na América Latina, a Advocacia em Saúde tem como objetivo principal lutar por melhores condições de vida e pela eqüidade. Isso significa desenvolver ações coletivas para obter direitos e combater as causas sociais da pobreza. As estratégias da Advocacia que têm tido mais impacto estão volta-

(16) UIPES - União Internacional de Promoção da Saúde e Educação para a Saúde. Editorial. Boletim, ano 2, n. 2, 2000.

(17) UNESCAP - United Nations Economic and Social Commission for Asia and Pacific. 
das para a obtenção de tratamento de Aids, controle do uso de fumo e drogas e proteção ambiental ${ }^{(18)}$.

Dentro de um Estado de Direito, não se pode falar em participação popular, empoderamento e eqüidade, sem legitimização. A Advocacia em Saúde é um caminho eficaz para agilizar a legitimização dos princípios essenciais elencados nas Cartas e Declarações e pela OMS, uma vez que, ela trata de problemas específicos, dá sustentação ao que a própria Constituição de 1988 e as Diretrizes do SUS preconizam, quanto ao processo de participação popular na definição e implementação de Políticas Públicas, abrangendo os espaços operativo, institucional e jurídico.

Assim sendo, há pela frente um processo coletivo (e de cada um) para: consolidar a redemocratização do país (e do Estado na Saúde); incentivar o exercício ativo da cidadania e ampliar o conceito e o campo de ação da Advocacia em Saúde, com a inclusão da PS. Ou seja, no âmbito da Advocacia em Saúde pode haver uma "Advocacia em Promoção da Saúde", com as mesmas estratégias acima alistadas, mas focalizada nos princípios e diretrizes da PS.

A Advocacia em Saúde é uma estratégia fundamental para a PS desenvolver seu papel político e suas diretrizes, e dar continuidade e sustentabilidade às suas políticas e práticas, considerando os diversos contextos e os diversos atores envolvidos. E a principal atividade da Advocacia em Promoção da Saúde seria trabalhar para incluir a Saúde e a PS na pauta dos outros setores sociais, o que por sua vez tornaria melhor definido o papel específico da própria área da Saúde na PS.

\section{REFERÊNCIAS BIBLIOGRÁFICAS}

BRASIL. Constituição da República Federativa do Brasil. Brasília, 1988.

BRASIL. Ministério da Saúde. Promoção da Saúde: Declaração de Alma-Ata, Carta de Ottawa, Declaração de Adelaide, Declaração de Sundsvall, Declaração de Santafé de Bogotá, Declaração de Jacarta, Rede de Megapaíses e Declaração de México. Brasília, 2001.

CERQUEIRA, M. T. Promoción de la salud y educación para la salud: retos y perspectivas. In: ORGANIZACIÓN MUNDIAL DE LA SALUD. La promoción de la salud y la educación para la salud en América Latina: un análisis sectorial. Genebra: Editorial de la Universidad de Puerto Rico, 1997. p. 7-48.

CONASEMS - Conselho Nacional de Secretários Municipais de Saúde. Pacto de saúde, Brasília, 2006. Disponível em: <www.conasems.org.br/files/dia27/ Of2ConstruindoMinisterioSaude02.pdf>. Acesso: maio 2007.

(18) WESTPHAL, M. F. et al. Public health policies and advocacy in Latin America: chances and environments to support these initiatives. Promotion \& Education, v. 7, n. 4, p. 29-32, 2000. 
DALLARI, S. G. Direito sanitário. In: MANUAL do Curso de Especialização em Direito Sanitário à Distância. Rio de Janeiro, 2002. p. 47-72.

et al. Advocacia em saúde no Brasil contemporâneo. Revista de Saúde Pública, São Paulo, v. 30, n. 6, p. 592-601, 1996.

DI PIETRO, M. S. Z. Direito administrativo. São Paulo: Atlas, 2000. p. 481-499.

MELLO, D. A. Reflexões sobre a promoção de saúde no contexto do Brasil. Cadernos de Saúde Pública, v. 16, n. 4, 2000.

PEREIRA, I. M. T. B. et al. Promoção da saúde e educação em saúde: uma parceria saudável. O Mundo da Saúde, ano 24, v.2 4, n. 1, p. 39-44, 2000.

SíCOLI, J. L.; NASCIMENTO, P. R. Promoção da saúde: concepções, princípios e operacionalização. Interface - Comunicação, Saúde, Educação, v. 7, n. 12, p. 101-22, 2003.

UIPES - União Internacional de Promoção da Saúde e Educação para a Saúde. Editorial. Boletim, ano 2, n. 2, 2000.

UIPES - União Internacional de Promoção da Saúde e Educação para a Saúde. Editorial. Boletim, ano 7, n. 9, 2005.

UNESCAP - United Nations Economic and Social Commission for Asia and the Pacific. Disponível em: <www.unescap.org/esid/hds/whatsnew/ 6GCHP_1.asp>. Acesso em: nov. 2005.

WESTPHAL, M. F. et al. Public health policies and advocacy in Latin America: chances and environments to support these initiatives. Promotion \& Education, v. 7, n. 4, p. 29-32, 2000.

WORLD HEALTH. Organization. Health promotion evaluation. Copenhagen, 1984.

WORLD HEALTH. Organization. Health promotion evaluation: recommendations to policymakers. Copenhagen, 1998. 